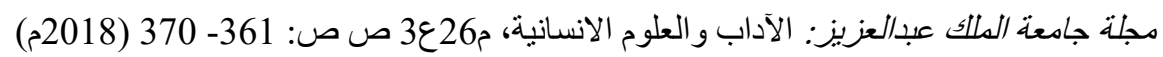

DOI:10.4197/Art.26-3.15

\title{
« La phrase arabe entre la théorie de l'analyse en constituants immédiats de Chomsky et la théorie dépendancielle de Tesnière »
}

\author{
Rabadi Najib and Alshammari Yaqoub \\ Université du Koweït \\ n.rabadi52@hotmail.com
}

\begin{abstract}
Résumé. Cette recherche étudie les convergences et les divergences dans l'application des courants linguistiques contemporains, (à savoir le modèle de l'Analyse en Constituants Immédiats et le modèle dépendanciel), sur la structure phrastique arabe, exprimée par la phrase verbale et la phrase nominale.

Mots-clés : Phrase arabe ; Stemma ; Arbre binaire ; Constituants immédiats ; Syntaxe ;

Abstract. This research studies the convergences and divergences in the application of the contemporary language currents (in the model of the Analysis in Immediate Constituents and the dependent model), on the Arabic phrastic structure, expressed by the verbal sentence and the nominal sentence.
\end{abstract}

Key words Arabic sentence, Stemma, binary tree, Immediate constituents, Syntax,

\section{Introduction}

Notre contribution a pour objet d'étudier la problématique concernant la place de la phrase arabe exprimée par ses deux types, traditionnellement appelés la phrase verbale, ğumla fi liyya, et la phrase nominale, ğumla ismiyya. Cette problématique sera abordée à la lumière des courants linguistiques contemporains, plus précisément le modèle dichotomique et syntagmatique de la grammaire générative, introduit par son précurseur, Noam Chomsky, d'un côté et, le modèle dépendanciel, introduit par son précurseur, Lucien Tesnière, de l'autre. Cet exposé essaie de montrer les convergences et les divergences dans l'application de ces deux modèles sur la structure phrastique arabe, à travers la représentation schématique de chaque modèle, à savoir l'arbre binaire pour le premier et le stemma pour le deuxième.

\section{Aperçu théorique}

De nos jours, nous constatons que chez beaucoup de linguistes contemporains, la conception aristo-platonienne sur la 
constitution du (logos) ou de la phrase décomposée en noms et en verbes, n'a guère changé. Tout au plus, les concepts onoma (ce dont on parle) + rhema (ce qu'on en dit) auraient pris différentes appellations selon la discipline envisagée: Sujet / Verbe, Sujet / Prédicat, Sujet / Attribut, Groupe nominal / Groupe verbal, Syntagme nominal / Syntagme verbal, etc... Les paires de Thème / Rhème, Topique / Commentaire, Support / Apport, ont plutôt un sens plus pragmatique. Nous devons ainsi la naissance de cette notion dichotomique à Platon et à Aristote, formalisée ultérieurement par Noam Chomsky en particulier.

En revanche, la notion de rection (gouvernement) et la notion de fonction introduites par les grammairiens-philosophes de Port-Royal (Arnauld Antoine et Lancelot Claude) en 1660, et avant eux, Sibawayhi, l'auteur d'Al-Kitāb, premier ouvrage de syntaxe arabe qui nous est arrivé, parlait déjà (in Sylvain, 2001 : 80) au VIII ${ }^{\text {ème }}$ siècle, du gouvernant et gouverné ${ }^{1}$ :

"Les grammairiens arabes du 8ième siècle, comme Sibawaih, distinguaient déjà gouverneur et gouverné en syntaxe et utilisaient cette distinction pour formuler des règles d'ordre des mots ou de rection " ${ }^{2}$. Owens 1988:79-81

Ainsi la notion de gouvernement et la notion de fonction, auxquelles s'ajoute la position centrale, attribuée au verbe dans l'énoncé par Condillac 1746, ont vraisemblablement préfiguré la conception dépendancielle, formalisée par la suite par Lucien Tesnière.

Cette littérature linguistique oppose aujourd'hui au moins trois études différentes portant sur l'analyse de la phrase: une

1 - Pièce maitresse dans l'histoire de la grammaire arabe, $A l$ Kitāb constitue un texte fondateur. Quant à son auteur, Sibawayhi mort en 177 du calendrier lunaire islamique (fin du VIII), il pose le premier matériel descriptif et systématique d'une grammaire de l'arabe.

${ }^{2}$ Cité par Sylvain Kahane 2001 dans son Introduction. conception dichotomique, une conception polytomique et une conception dépendancielle. Ces courants linguistiques divergent dans le découpage syntaxique de l'unité phrastique et, par conséquent, ne donnent pas les mêmes résultats, comme nous allons le montrer dans les pages qui viennent.

La conception polytomique qu'est le fonctionnalisme (le fonctionnalisme d'A. Martinet en particulier), aurait la possibilité d'être ramenée à l'un ou à l'autre courant. $\mathrm{Vu}$ son adoption de la description, dite structurale et de la composition de l'énoncé en deux éléments obligatoires, à savoir le sujet et le prédicat, (même si le complément du verbe est parfois considéré comme une expansion, susceptible d'être supprimée), ce courant pourrait être rapproché à l'analyse en constituants immédiats. Cependant, nous le trouvons plus proche de la théorie de dépendance car d'un côté, Martinet (1960), lui-même, trouve que les critères de la «distribution» et de la «transformation», introduits par la théorie syntagmatique dichotomique, ne sont pas suffisants pour la description d'une langue: «Il (Martinet) critique l'idée de la distribution comme étant un critère insuffisant pour bâtir une description de la langue. La transformation non plus : elle ne peut pas expliquer, sans un informateur, ni la raison des transformations, ni leur justesse », c'est nous qui soulignons, (Dina Kassas, 2005 : 36). Notons au passage que la syntaxe transformationnelle est née à cause des problèmes que la syntaxe distributionnelle ne parvient pas à résoudre. D'un autre côté, le principe même de la dépendance est clair chez les fonctionnalistes ; Guérin Françoise 82-83 le confirme dans ce passage :

"Une fonction syntaxique sera le rapport qui est établi dans l'énoncé entre deux monèmes appartenant à des classes compatibles tel qu'un monème est déterminant de l'autre et qu'il existe entre les deux monèmes un 
rapport de subordination. Une fonction grammaticale est une relation à sens unique qui s'établit de déterminant à déterminé, $d u$ subordonné vers un élément plus central, ou, en d'autres termes, d'une expansion vers un noyau ».

Tesnière (1988:39) insiste sur cet aspect fonctionnel qui constitue avec l'aspect syntaxique l'essence même de sa théorie : il ne peut $\mathrm{y}$ avoir structure qu'autant qu'il y a fonction et,... la syntaxe structurale est en même temps la syntaxe fonctionnelle ». Alors que la pratique des constituants immédiats attribue à la fonction une place secondaire, moins importante que la syntaxe. Pour notre part, nous essayerons d'appliquer les deux modèles, à savoir le modèle syntagmatique dichotomique et le modèle dépendanciel, à la phrase arabe. Mais avant, il nous faut identifier de quels types de phrase dont il s'agit.

\section{La phrase en arabe}

Les langues naturelles se classent en catégories selon leur système syntaxique et leur propriété morphologique qui se manifestent dans l'unité phrastique. Ainsi Steele (1978) divise les langues en trois catégories :

«... les langues à ordre rigide ou syntaxiquement contraintes comme l'anglais, les langues à ordre libre ou communicativement contraintes comme le finlandais, et les langues à ordre mixte », ... «Si l'on emploie la terminologie de Steele, l'arabe se classe comme une langue à ordre mixte. ", cité par D. Kassas, p.269.

Ces trois catégories correspondent respectivement aux langues, dites configurationnelles (l'anglais, le français), aux langues non-configurationnelles (le latin), et aux langues semi-configurationnelles (l'arabe). L'arabe est donc une langue à ordre mixte ou semi-configurationnelle: parce qu'elle est morphologiquement riche et nous présente plusieurs structures phrastiques plus ou moins ordonnées.

Le champ de notre recherche se restreint à la distinction de la grammaire traditionnelle entre " phrase verbale» et " phrase nominale». Selon cette grammaire, est nominale la phrase commençant par un nom. Est verbale la phrase commençant par un verbe. Bon nombre de grammairiens trouvent cette distinction non pertinente.

Certains linguistes arabes, tels que, Ahmad Abd al-Sattār Al-Jawari, Mahdi Al-Makhzūmī, Al-Samarra'ī, entre autres, ne trouvent pas de différence entre les deux phrases: la phrase, dite nominale, n'est, pour eux, qu'une phrase verbale, étant donné que le sujet et le prédicat sont respectivement les mêmes dans les deux structures phrastiques. Pour ces linguistes, la nature de la prédication est la même dans les deux phrases et, l'emplacement initial du sujet n'a de raison d'être que pour la focalisation de ce dernier. Al-Jawārī le confirme dans cette citation en langue arabe, empruntée à (Salah eddine Al-Za'balawi 1991) :

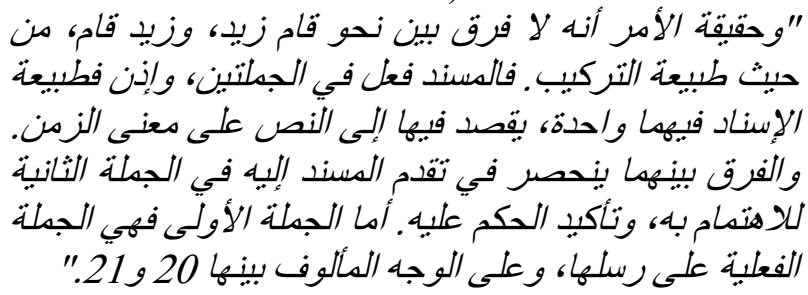

Il est à rappeler aussi que cette position a été déjà adoptée par les grammairiens arabes du courant Al-Kūfa, comme le montre le passage suivant :

"وهكذا برى الأستاذ الجواري أن تقديم الفاعل بالابتداء لو لوباء

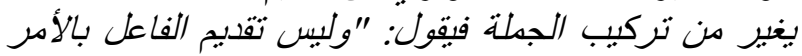

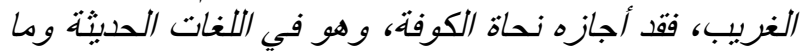

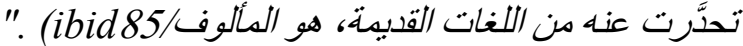
Abou Al-Makaarim Ali (2007:56) s'aligne à ces grammairiens en adoptant la même conception à propos de la non-pertinence de cette distinction. Pour lui, cette non-pertinence relève d'un fait fonctionnel: la fonction syntaxique du nom tête de la phrase nominale est mubtada? (inchoatif), tandis que la fonction syntaxique du nom postposé dans la phrase verbale est fâ-il (agent). Le problème réside donc dans l'antéposition et la postposition du nom dans ces deux types de phrases. Pour lever cette ambiguïté de fonction, Abou Al-Makaarim rejette cette distinction traditionnelle et considère que la phrase dont le prédicat est un verbe sera 
phrase verbale quel que soit l'emplacement du verbe :

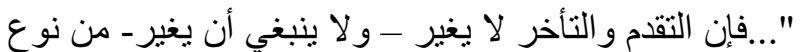

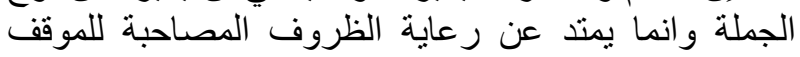

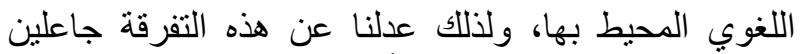

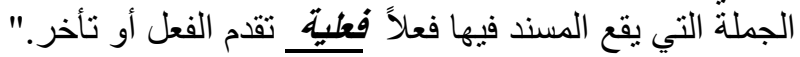
(56:2007) Ainsi, il fait fondre la phrase nominale dans la phrase verbale. Un autre courant, en revanche, tout en partageant l'opinion des linguistes déjà mentionnés ci-dessus, à propos de cette non pertinence, opte pour une position inverse : il s'agit, cette fois, de la fusion de la phrase verbale dans la phrase nominale.

Ce courant est représenté par des linguistes, tels que 'Awwad (1973), Shehadeh (1986), Harroum (1989), qui trouvent que l'ordre canonique des phrases verbales arabes (VSO) doit être obtenu à partir d'une transformation permettant le déplacement du verbe avec ses auxiliaires... et la phrase arabe s'analyse en base en un groupe nominal suivi d'un groupe verbal, note A. Ben Gharbia (2011). Leur argument principal peut être ramené au principe selon lequel une prédication doit être constituée d'un sujet et d'un prédicat quel que soit l'ordre de mots.

D'autres linguistes vont plus loin en dissolvant la phrase verbale (VSO) dans la phrase nominale (SVO) pour justifier leur croyance en une grammaire universelle :

"Dans le cadre de la grammaire universelle (GU), Fehri (1982 et 1993) élabore une description interprétative de la structure de la phrase en ASM (arabe standard moderne). Dans l'approche $G U$, interprétative signifie expliquer les faits d'une langue en vue d'esquisser le système grammatical sous-jacent. La description se base sur l'existence d'une asymétrie d'accord entre un ordre SVO et un ordre standard non marqué VSO et propose un ensemble de règles de montée pour accommoder l'ordre VSO dans une séquence SVO». Cité par El Kassas 2005: 49, qui ajoute que "l'approche $G U$ suppose l'existence d'un ordre SVO universellement reconnu par toutes les langues naturelles. Nous ne pensons pas que cela soit vraiment le cas : beaucoup de langues démentent cette supposition; ".

D'autres encore qui se réclament de la linguistique d'inspiration fonctionnaliste, affirment que l'ordre canonique n'est pas VSO, mais plutôt SVO, car c'est le verbe qui dépend du sujet et non pas l'inverse. Leur argument se base sur l'antériorité existentielle du nom sur le verbe. Ibn Ğinnī écrit à ce propos: «Les noms sont les premiers et les verbes en dépendent », ( Al-Khassais, tome 3, p. 84). Les fonctionnalistes et en particulier les générativistes, adoptent plus ou moins la même opinion en donnant la priorité au nom.

Notre propos ne s'accorde ni avec les uns ni avec les autres. Nous pensons qu'au lieu de dissoudre la phrase verbale dans la phrase nominale ou l'inverse, il serait plus pertinent de chercher l'individualité de chacune de ces deux phrases qui, à notre avis, constituent deux structures phrastiques autonomes et distinctes ; c'est ce que nous allons essayer de montrer dans les lignes qui viennent.

\section{La phrase verbale et la phrase nominale}

Traditionnellement parlant, la phrase verbale et la phrase nominale, deux structures phrastiques coexistent et sont d'usage dans le système linguistique de la langue arabe. Encore une fois, selon cette tradition grammaticale, est nominale la phrase commençant par un nom suivi d'un verbe ; et est verbale la phrase commençant par un verbe suivi de son agent. Nous allons essayer de montrer les particularités morphologique, fonctionnelle, sémantique, syntaxique, etc... de ces deux types de phrase. Analysons ces deux exemples :

1- Al-waladu ğà'a

L'enfant vient. $\quad=$ phrase nominale

2. ğā'a al-waladu

Vient l'enfant = phrase verbale

- particularité morphologique: 
Le verbe dans la phrase verbale est toujours au singulier quel que soit le nombre de l'agent :

-ğà'a al-waladu (vient l'enfant)

-ğā'a al-waladān (vinrent les deux enfants)

-ğà'a al-awalādu (vinrent les enfants).

Dans la phrase nominale, le verbe change de forme :

- Al-waladān ğā'ā (les deux enfants vinrent)

-Al-awlādu ğì' $\bar{u}$ (les enfants vinrent).

Ici, nous constatons que la morphologie du verbe change en fonction du nombre du sujet, s'il est duel ou pluriel.

- particularité fonctionnelle :

al-waladu dans la phrase verbale (ex .2, cidessus) est l'agent du verbe ğāa'a, mais alwaladu dans la phrase nominale (ex.1, cidessus) n'est pas l'agent, il a la fonction de mubtada? (inchoatif) et le verbe ğā'a est habar (attribut).

- particularité de l'emploi :

Nous avons pu relever quelques situations qui favorisent plutôt l'emploi de la phrase nominale : les slogans, les titres de la presse et la traduction simultanée.

- « Le slogan, selon O. Reboul (1975 :28), est une formule concise et frappante, ..., destinée à faire agir les masses tant par son style que par l'élément d'autojustification, ... ", (C'est nous qui soulignons). Les circonstances discursives $\mathrm{du}$ slogan privilégient vraisemblablement l'emploi de la phrase nominale. Car cet emploi situationnel nous pousserait à croire qu'elle est beaucoup plus proche des faits énonciatifs, communicationnels que la phrase verbale. Sylvain Kahane (2001: 55) conclut son exposé dans ce sens et confirme que « $L a$ structure communicative joue un rôle essentiel dans la hiérarchisation du graphe sémantique (notamment le choix de la tête syntaxique de la phrase) et dans la linéarisation. Dans les langues à ordre des mots relativement libre comme le russe ou l'allemand, la structure communicative (notamment la partition thème rhème et la focalisation) contrôle fortement l'ordre des mots et la prosodie. "।

Pour construire un slogan, on s'investit plutôt dans la phrase nominale:

démocratie)

$$
\text { (le peuple veut la }
$$

départ du régime)

(le peuple veut la chute du régime)

Pour les titres de la presse, nous avons réuni un ensemble d'énoncés réels représentant des informations et transmis sous forme de titres au téléjournal. Ces énoncés qui sont liés au discours direct, sont tous exprimés par des phrases nominales. En voici quelques exemples authentiques :

-مستوطنون يقتحمن المسجد الأقصى : Des colons assaillent la Mosquée Al-Aqsa.

Les forces de l'armée occupante agressent les manifestants. Le ministre de l'intérieur présente sa démission. Moscou condamne les attentats en Arabie Saoudite. =Les =-المسلمون يحتفلون بعيد الفطر السعيد

\section{Musulmans fêtent le I'd Al-fider.}

Pour la traduction simultanée d'une langue à ordre rigide vers une langue à ordre mixte, l'emploi de la phrase nominale devient souvent obligatoire. Il n'est pas du tout pertinent, par exemple, d'attendre l'apparition (l'écoute) du verbe reçoit dans cette phrase française : Le directeur de la Société Nationale des Chemins de Fer Française, Monsieur X. $Y$., qui vient d'être nommé à la tête de l'établissement, recoit les délégués syndicaux, pour commencer à traduire un tel syntagme nominal aussi riche d'éléments et d'expansions. Il est donc indispensable de la traduire par une phrase nominale en commençant directement par le syntagme sujet.

A ces particularités, nous pouvons également ajouter d'autres, tel que l'aspect modal, c'està-dire si le locuteur a l'intention de mettre le 
nom en relief, il dira (al-waladu ğā'a) et s'il a l'intention d'insister sur l'action, il dira ( ̌̆ā'a al-waladu). Le locuteur commence donc par le contenu sémantique auquel il attribue plus d'importance. Du point de vue fréquence, il est claire que la phrase verbale est beaucoup plus utilisée que la nominale.

- particularité syntaxique :

Cette particularité syntaxique que nous allons analyser ci-après, nous permet de voir clairement les différences entre les deux phrases en question. Commençons d'abord par la nominale.

Soit la phrase nominale suivante ${ }^{3}$ : الولد يرسل (l'enfant lance la balle)

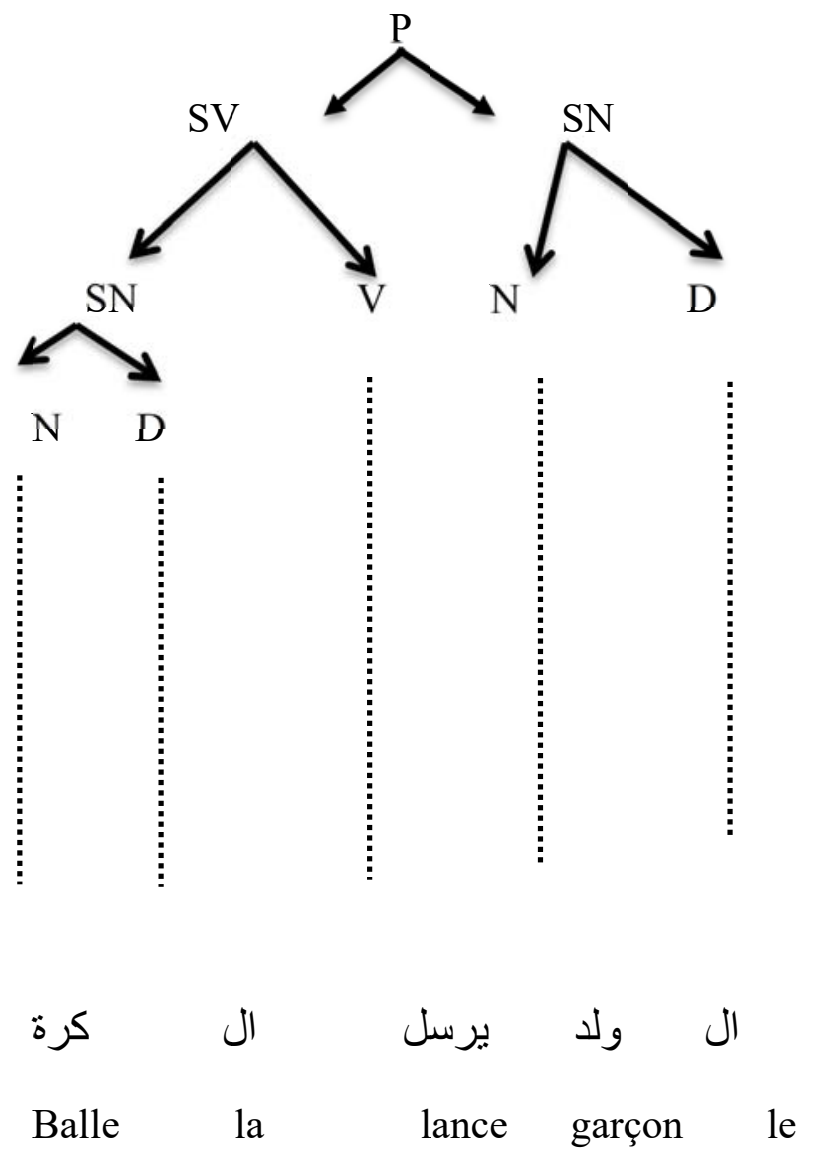

3 - Il est à rappeler que l'arabe s'écrit de droite à gauche.
Nous rappelons que l'Analyse en Constituants Immédiats divise la phrase nominale en deux grands ensembles: le syntagme nominal (SN) et le syntagme verbal (SV). Ces deux syntagmes représentent ce qu'on appelle les Constituants Immédiats de la phrase (P). Ils se décomposent à leur tour en d'autres constituants pour finir avec les unités ultimes qu'on ne peut plus découper en d'autres plus petites. Ce modèle procède donc en groupes ou plus précisément en syntagmes. L'analyse en constituants est horizontale: l'indicateur syntagmatique décompose la phrase en ses plus grands constituants, puis en constituants inférieurs jusqu'à ce que l'analyse atteigne les ال- ولد برسل ال- بر ): constituants ultimes كرة sont contenus dans d'autres constituants. Ce courant dichotomique a pour but de montrer les rapports syntaxiques que les constituants de la phrase entretiennent entre eux. Le Dictionnaire de linguistique et des sciences du langage, Larousse, définit cette théorie de la manière suivante: "La théorie des constituants en décrit la structure comme la combinaison (concaténation) de deux constituants: un syntagme nominal (l'enfant) et un syntagme verbal (lance la balle). Chacun de ces deux constituants immédiats de la phrase, est à son tour, formé de constituants. " (p. 114).

Nous rappelons que, malgré les aménagements divers effectués sur les théories de Chomsky: la grammaire de réécriture syntagmatique (GRS), la grammaire générative et transformationnelle (GGT), la grammaire générative et transformationnelle standard (GGT standard) et la théorie, dite X-barre, le principe du point de départ de l'Analyse en Constituants Immédiats de la phrase, basé sur la dichotomie (noun phrase et verbal phrase), n'a pas été affecté.

En revanche la thèse de Tesnière découpe la phrase en mots isolés pour repérer les liens 
syntaxiques entre eux. Pour ce faire, il utilise une représentation graphique qu'il appelle "stemma" :

Soit la phrase verbale suivante: الكرة "الولد (l'enfant lance la balle)

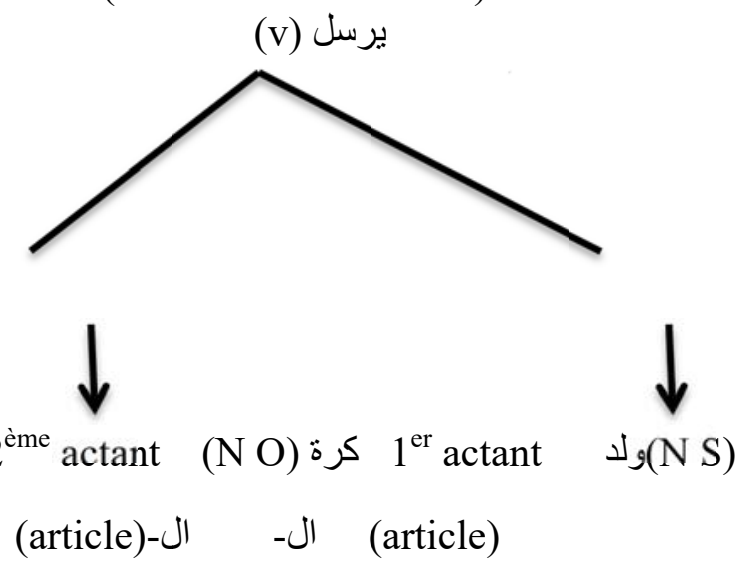

Il s'agit d'une représentation schématique des relations entre les éléments de la phrase. Sur ce stemma, les relations entre les éléments de la phrase sont verticales: un nucleus (une partie du discours) en régit un autre. Le supérieur est le noud régissant et l'inférieur est le noud régi. Ainsi le verbe (يرسل) dans l'exemple ci-dessus, est le régissant de l'agent (ولد) et de l'objet (كرة) qui sont les régis. Dans le même temps, (ولد) (كرة) régissent respectivement les déterminants (al) et (al). Tesnière appelle cette relation dépendance. Il est important de répéter que, selon cette théorie, le verbe occupe la place centrale dans la phrase. C'est pour cela qu'il a été mis au sommet de la pyramide du diagramme. C'est lui qui organise la structure de la phrase et distribue les fonctions des éléments de son entourage. N'ayant pas, lui-même, de fonction syntaxique, le verbe représente le procès d'une pièce de théâtre ( $\mathrm{si}$ on compare cette dernière à la phrase), l'agent et l'objet qui sont les acteurs, représentent respectivement le $1^{\text {ier }}$
Actant et le $2^{\text {ième }}$ Actant, selon les termes de Tesnière ${ }^{4}$.

Cette analyse dépendancielle procède en mots isolés pour identifier de près les rapports hiérarchiques se manifestant verticalement entre eux sur le stemma. Le déterminant, par exemple, détermine uniquement le nom auquel il se rapporte et non tout le syntagme. Cette place primordiale donnée au verbe par ce courant dépendanciel hiérarchique, nous fait croire qu'on est proche de la phrase canonique arabe qu'est la phrase verbale ayant comme ordre VSO. Ceci est confirmé par Sylvain Kahane (2001: 48) :

"Bien que la grammaire arabe classique se présente comme une grammaire de dépendance, on ne trouve pas actuellement beaucoup de travaux sur l'arabe dans le cadre des DG. Ceci est peut-être dî au fait que les DG n'ont pas témoigné du même essor que les PSG. La quasi-totalité des travaux récents sur la syntaxe de la langue arabe se classent dans le cadre des grammaires syntagmatiques. ». C'est nous qui soulignons.

Comme nous pouvons le constater, la phrase nominale répond aux propriétés de la théorie syntagmatique quant à la dichotomie et, la phrase verbale répond à celle de la théorie dépendancielle quant à la position hiérarchique et privilégiée du verbe. Les divergences entre ces deux modèles sont cependant fondamentales. Essayons maintenant

\footnotetext{
4 - Contrairement à ce que l'on croit souvent, la comparaison de la proposition à une scène de théâtre ne serait pas une idée de Tesnière: "Une proposition, c'est comme une scène où chaque terme joue son rôle. L'un fait l'action : c'est le sujet; l'autre la supporte, c'est l'objet; d'autres termes expriment le moment où l'action se fait, ou bien expriment le lieu, la manière....». Maquet, Flot et Roy, Cours de Langue Française, Cours moyen et supérieur, Hachette, Paris, 1ièr éd. 1925.( Cité par Najib Rabadi, Thèse de doctorat, 1997, p .181)
} 
d'analyser les deux types de phrase à la lumière de ces deux modèles.

\section{Convergence et divergence d'application}

Le précurseur de la théorie syntagmatique a appliqué son modèle uniquement sur la phrase canonique anglaise (SVO), alors que Tesnière a expérimenté le sien sur plus de soixante langues. La phrase nominale arabe, comme nous venons de voir, s'analyse parfaitement au modèle syntagmatique sans problèmes, parce que la structuration positionnelle des éléments constitutifs de cette phrase ressemble à celle de la phrase canonique anglaise: elle commence par un nom sujet suivi du verbe et son objet par la suite.

Par ailleurs, nous avons du mal à appliquer le modèle dichotomique de Chomsky sur la phrase verbale arabe tout simplement à cause de son ordre standard (VSO) : elle commence par un verbe suivi directement de son agent et ensuite son objet.

Ainsi, si l'on suit l'analyse formelle du courant syntagmatique sur la phrase verbale, il s'avère que le Constituant Immédiat de la phrase, l'agent, tombe entre le verbe et son objet, qui tous les deux, constituent le deuxième Constituant Immédiat, le prédicat, et qui devraient former ensemble un syntagme, c'està-dire une seule unité syntaxique. Le fait d'intercaler l'agent entre le verbe et l'objet, divise l'unité syntaxique du syntagme verbal, et par conséquent, perturbe et déforme la notion même de syntagme. Cette procédure nous semble donc épineuse dans la mesure où ce modèle même postule la division dichotomique de la phrase en partie Sujet et partie Prédicat. Le problème de ce modèle s'aggrave dans son application sur des langues à ordre libre comme le latin, le russe, le slovène qui n'ont pas d'ordre syntaxique ordonné; on peut dire en latin par exemple : Filius amat patrem, patrem amat filius, amat filius patrem, amat patrem filius, filius patrem amat, patrem filius amat. Quel que soit l'ordre des mots, cette phrase latine signifie respectivement en français et en anglais : le fils aime son père; the son likes his father. La division dichotomique n'est donc pas pertinente dans les langues casuelles.

De surcroît, pour pouvoir résoudre des faits linguistiques qui vont à l'encontre de cette théorie et qui se trouvent même dans les langues à ordre rigide, le modèle dichotomique nous propose de chercher des procédures de transformation. Pour notre cas, nous n'avons pas besoin de faire appel à une telle procédure car la langue arabe dispose déjà de ce type de structure, à savoir la phrase nominale. Le fait de transformer la phrase verbale, conventionnellement reconnue de phrase canonique en arabe, en phrase nominale pour l'adapter au modèle syntagmatique, nous semble inapproprié. La phrase verbale en arabe n'a apparemment pas eu ce qu'elle mériterait pour qu'elle soit étudiée en tant que telle par les linguistes. Cependant le modèle dépendanciel nous dispense de faire appel à des opérations transformationnelles, car il couvre aussi bien les langues à ordre rigide que les langues à ordre libre ou même les langues à ordre mixte. Le stemma (issu de ce modèle) assimile les deux structures phrastiques en question. 


\section{Conclusion}

Nous avons essayé de décrire un aspect syntaxique de la structure phrastique en arabe. Il s'agit des deux phrases arabes, conventionnellement connues sous le nom de phrase nominale et phrase verbale et, après avoir présenté leurs propriétés, nous les avons analysées sur le schéma de l'Analyse en Constituants Immédiats de la grammaire syntagmatique et sur celui de la théorie de dépendance. Nous avons trouvé que le modèle syntagmatique dispose de rapports symétriques applicables à la nominale seule quant à l'ordre des mots et à la structuration dichotomique de la phrase en syntagme nominale et syntagme verbal; alors qu'il dispose des rapports asymétriques et non applicables à la verbale. Le modèle dépendanciel, en revanche, assimile bien les deux phrases (nominale et verbale). Cette application nous a permis de montrer que le génie de la langue arabe peut absorber les deux théories sans soumettre ses faits linguistiques à des opérations transformationnelles pour les adapter à un modèle ou à un autre. Nous espérons que cette modeste contribution ouvrirait des pistes nouvelles dans la recherche de la structure phrastique de la langue arabe.

\section{Bibliographie sélective}

Ben Gharbia Abdeljabbar, 2011, «Les schémas de phrase en arabe et en français », Syntaxe et sémantique, $\mathrm{N}^{\circ} 12$, p. 49-72.

Dina EL KASSAS, 2005, « Linguistique théorique, descriptive, automatique. Une étude contrastive de l'arabe et du français dans une perspective de génération multilingue », thèse de doctorat Université Paris 7.
Guérin Françoise, 2009, « Les fonctions

syntaxiques dans la théorie fonctionnaliste d'André Martinet », La linguistique, Vol. 45, p. 81-86.

Guillaume J.-P., 1986, « Sibawayhi et

l'énonciation : une proposition de lecture », P. 5362 Histoire, Epistémologie, Langage.

J. Dubois, M. Giacomo, L. Guespin L., Ch. J-B. Marcellesi , J-P. Mevel. 1994, Larousse, dictionnaire de linguistique et des sciences $d u$ langage. Paris : Larousse.

Maquet, Flot et Roy, 1925, « Cours de Langue Française, Cours moyen et supérieur ", Hachette, Paris, 1 ièr éd.

N. Rabadi, 1997, "La notion de constituant en syntaxe $d u$ français. L'interaction des théories linguistiques et des grammaires scolaires de 1843 à nos jours ", thèse doctorat, Université Lumière Lyon II.

Sylvain Kahane, 2001, « Grammaires de dépendance formelles et théorie Sens-Texte1 », TALN, Tours, 2-5 (version revue et corrigée, juillet 2001), Université Paris 7.

TESNIERE, Lucien, 1988, «Elément de syntaxe

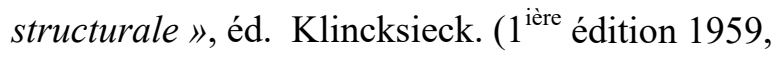
1988 :39).

Reboul Olivier, 1975, « Le Slogan » éd. complexe, Bruxelles.

\section{- Référence arabe:}

- Ibn Jinny, "Al-Khassais », tome 3, p. 84.

$$
\begin{aligned}
& \text { علي أبو المكارم، } 2007 \text { ، الجملة ألأسمية ، مؤسسة المختار للنشر }
\end{aligned}
$$

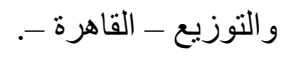

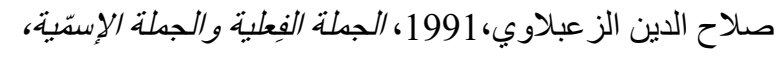

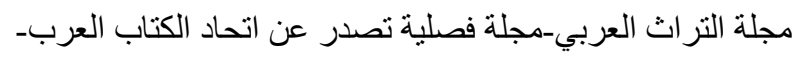

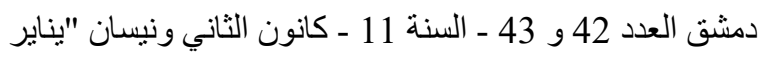

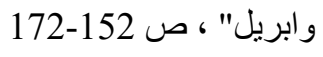

Salah eddine Al-Za'balawi, 2001, «La phrase verbale et la phrase nominale ", Majalat Altourath Al-a'rabi, Damas.No 42\&43,153 -172. 
الجملة العربية بين نظرية المكونات المباثرة لثومسكي

\section{ونظرية التبعيّة لتينبير}

نجيب الربضي و يعقوب الثمري

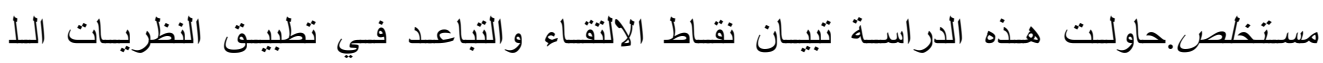

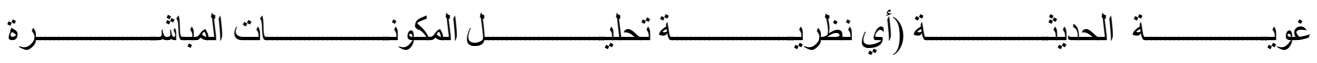

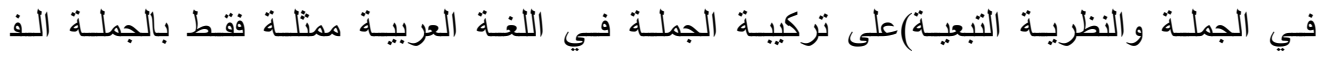
علية والجملة الاسمية.

كلمات دالة: الجملة العربية ـ ستيما ـ الشجرة البينية ـ المكوّن المباشر ـ 